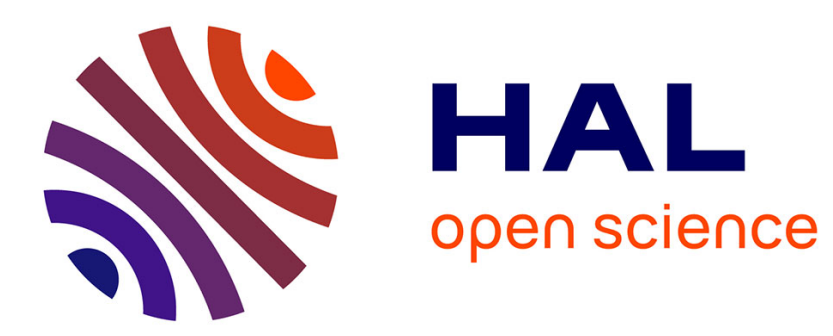

\title{
Sensitivity of the liquid bridge hydrodynamics to local capillary contributions
}

\author{
Eric Chénier, C. Delcarte, G. Kasperski, G. Labrosse
}

\section{To cite this version:}

Eric Chénier, C. Delcarte, G. Kasperski, G. Labrosse. Sensitivity of the liquid bridge hydrodynamics to local capillary contributions. Physics of Fluids, 2002, 14 (9), pp.3109-3117. 10.1063/1.1490350 . hal-00693612

\section{HAL Id: hal-00693612 https://hal.science/hal-00693612}

Submitted on 12 Jun 2017

HAL is a multi-disciplinary open access archive for the deposit and dissemination of scientific research documents, whether they are published or not. The documents may come from teaching and research institutions in France or abroad, or from public or private research centers.
L'archive ouverte pluridisciplinaire HAL, est destinée au dépôt et à la diffusion de documents scientifiques de niveau recherche, publiés ou non, émanant des établissements d'enseignement et de recherche français ou étrangers, des laboratoires publics ou privés. 


\title{
Sensitivity of the liquid bridge hydrodynamics to local capillary contributions
}

\author{
E. Chénier ${ }^{\dagger}$, C. Delcarte ${ }^{\ddagger}$, G. Kasperski ${ }^{\ddagger}$, G. Labrosse $e^{\ddagger}$ \\ †Uiversité de Marne-la-Vallée, LETEM, 77454 Marne-la-Vallée Cedex 2, France \\ * Université Paris-Sud XI, LIMSI-CNRS, Bât. 508, 91405 Orsay Cedex, France
}

(October 2, 2001)

\begin{abstract}
In the usual models of thermocapillary flows a vorticity singularity occurs at the contact free surface / solid boundaries. The steady axisymmetric hydrodynamics of the side-heated liquid bridge of molten metal is addressed here for its sensitivity to the size $\delta$ of a length scale explicitly introduced to regularize the problem. By a linear stability analysis of the flows, various stable steady states are predicted, the already known ones which are self-symmetric by reflection about the mid-plane, but also others which do not enjoy this property. The thresholds in $M a$ of the associated bifurcations are strongly dependent on $\delta$, and converge with $\delta \rightarrow 0$ towards low values. Published data give these results some physical relevance. Some elements are proposed on the physics of the symmetrical states loss of stability.
\end{abstract}

\section{INTRODUCTION}

The floating zone technique $[1,2]$ is widely used for manufacturing high quality single crystals. In this process, a melted zone is created between two co-axial poly- and mono-crystalline cylindrical rods by means of an axisymmetric lateral heating, and slowly displaced towards the poly-crystalline rod so that the liquid re-solidifies with a monocrystalline structure. Being maintained by surface tension, the molten phase cannot be contaminated by the crucible. It is well known that the resulting crystals can exhibit defaults induced by unsteady convection occurring within the liquid bridge. The study of the mechanisms governing the flow dynamics and the heat transfers is then particularly helpful for controlling the crystal growth process and thereby the quality of its products.

Numerous approaches and models have been employed to progress in the understanding of this physical configuration. For the experimentalist the access to measurements in the core of the flow is limited to transparent fluids, having a Prandtl number $(\mathrm{Pr})$ value larger than 6 or so. Numerical simulations can bring valuable contributions, but the complexity of the physics generates other limitations, such as non-deformable free surface, absence of phase change kinetics, or also reduction of the flow dimensionality (essentially 2D so far [3]). In this spirit of simplifying for performing detailed studies, the half-zone model has been initially conceived as a rough approximation of the sideheated liquid bridge, assuming that the flow in this latter configuration enjoys the property of reflection symmetry about the "horizontal" mid-plane (П). An other aspect of the physical complexity of the capillarity driven flows lies in the modeling of the contact of the free surface with the solid boundaries. Here again it is judicious to simplify the physics by discarding, in a first approach, any solidification process. Then it remains the ticklish problem of the singularity generated by the usual hydrodynamic considerations, that most of the numerical works simply hasten to circumvent by filtering it out, one way or another.

The present paper addresses this contact singularity problem for a side-heated liquid bridge model. Regularizing explicitly the singularity and following the evolution of the numerical flows and the convergence of their stability thresholds with a new free parameter (the filter's length scale) leads to an important conclusion : the global structure of the flow is sensitive to local contributions of the capillary forces. Another important consequence is that the stable steady axisymmetric flows can also be non-symmetrical by reflection with respect to $\Pi$, at small Marangoni number $(M a)$ values. In our simplified model, the free surface is non-deformable, and the flow is two-dimensional. But the presence of the singularity is not related with those simplifications. A physically relevant modeling of the contact free surface / solid boundary appears therefore as a pre-requisite of any numerical simulation, before tackling threedimensional configurations, with or without deformable free surfaces. 


\section{THE PHYSICAL MODEL}

Let us consider the floating zone model presented in Fig. 1. Gravity is absent. The axisymmetric flow is described in a $(O, r, z)$ radial and axial coordinate system, with $\boldsymbol{e}_{r}$ and $\boldsymbol{e}_{z}$ the corresponding unit vectors. The origin $O$ is located at the center of the full liquid bridge. The cylindrical liquid bridge, of radius $R$ and length $2 R$, is held by surface tension between two solid planar and coaxial disks of radius $R$, maintained at the uniform temperature $T_{m}$. The free surface is straight and submitted to a steady heating flux $Q=Q_{0} q(z)$, with $Q_{0}$ the maximum heat flux density. The non-dimensional flux $q(z)$ is symmetrical about the mid-plane $I$ defined by $z=0$ and it must cancel at both extremities $(z= \pm 1)$ for preserving the disks temperature uniformity. It is given the polynomial form [2,4] $q(z)=\left(1-z^{2}\right)^{2}$. The capillary convective flows are governed by the Navier-Stokes and energy equations, of which the Boussinesq non-dimensional expression reads:

$$
\begin{aligned}
\frac{\partial u}{\partial t}+(u \cdot \nabla) u & =-\nabla p+\operatorname{Pr}\left(\nabla^{2}-\frac{e_{r}}{r^{2}}\right) u, \\
\frac{\partial \theta}{\partial t}+u \cdot \nabla \theta & =\nabla^{2} \theta, \\
\nabla . u & =0 .
\end{aligned}
$$

The operators are $\nabla=\boldsymbol{e}_{r}(\partial / \partial r)+\boldsymbol{e}_{z}(\partial / \partial z)$ and $\nabla^{2}=(1 / r)(\partial / \partial r)[(r(\partial / \partial r))]+\partial^{2} / \partial z^{2}$. Noting $\boldsymbol{u}=v \boldsymbol{e}_{r}+w \boldsymbol{e}_{z}$, one has also $\nabla \cdot u=(1 / r)[\partial(r v) / \partial r]+(\partial w / \partial z)$ and $u \cdot \nabla=v(\partial / \partial r)+w(\partial / \partial z)$. The length, temperature, velocity, pressure and time scales respectively are $R, \Delta T=Q_{0} R / \lambda, U=\kappa / R, \rho U^{2}$ and $R / U$, where $\rho$ and $\lambda, \kappa$ are the fluid density and thermal conductivity, diffusivity. The reduced temperature is $\theta=\left(T-T_{m}\right) / \Delta T$. Then, non-dimensional parameters are introduced, the Marangoni $\left(M a=\left[-\left.(\partial \sigma / \partial T)\right|_{T_{m}} R / \mu \kappa\right] \Delta T\right)$ and Prandtl $(\operatorname{Pr}=\nu / \kappa)$ numbers, where $\sigma$, and $\mu, \nu$, respectively stand for the surface tension and dynamic, kinematic viscosity. The Prandtl number is from now on fixed, $\operatorname{Pr}=10^{-2}$.

To complete the set ((1)-(3)), boundary conditions are specified:

$$
\begin{aligned}
& \cdot z= \pm 1\left\{\begin{array}{l}
u=0 \text { (no-slip conditions), } \\
\theta=0 \text { (imposed temperature), }
\end{array}\right. \\
& \cdot r=1 \quad\left\{\begin{array}{l}
v=0 \text { (non-deformable free surface), } \\
\frac{\partial w}{\partial r}=-M a \frac{\partial \theta}{\partial z} \text { (stress condition), } \\
\frac{\partial \theta}{\partial r}=q(z) \text { (heat flux). }
\end{array}\right. \\
& \cdot r=0\left\{\begin{array}{l}
v=0, \\
\frac{\partial w}{\partial r}=0 \text { (axial symmetry conditions), } \\
\frac{\partial \theta}{\partial r}=0 .
\end{array}\right.
\end{aligned}
$$

\section{THE SINGULARITY}

The boundary conditions for $w$ and $\theta$ at $r=1$ are of flux type and must match the $z= \pm 1$ conditions for the problem to be well posed. For instance, as already indicated, the heat flux $\left.\left(\frac{\partial \theta}{\partial r}\right)\right|_{r=1}$ must vanish at the ends $z= \pm 1$ where $\theta$ is constant with $r$. The same argument holds for $\left.\left(\frac{\partial w}{\partial r}\right)\right|_{r=1}$, which implies that $\left.\left(\frac{\partial \theta}{\partial z}\right)\right|_{r=1, z= \pm 1}$ should cancel. This is certainly not satisfied, in fact less and less as Ma increases. Indeed, the heat supplied laterally is convected by the flow towards both solid disks where it penetrates by conduction, that is with $\left.\left(\frac{\partial \theta}{\partial z}\right)\right|_{z=+1} \neq 0$. Obviously, this conduction flux is larger (in absolute value) at the contact of the free surface with the disks than elsewhere along them. Since $\left.\left(\frac{\partial w}{\partial r}\right)\right|_{r=1}=\omega(r=1, z)$ is the azimuthal vorticity along the free surface, the model presents a vorticity singularity. It is, in fact, inherent to the association of capillarity and no-slip conditions at the junction free surface / solid boundary, and not related to the geometrical simplification of our model. Regardless of its shape (Fig. 2), the free 
surface is source of positive azimuthal vorticity in the upper part of the cavity, until its extremity, whilst adherence on the solid boundary generates negative vorticity. This vorticity singularity might have much to do with the velocity singularity encountered along the moving contact lines. The analysis of this configuration is still subject of research if one refers for instance to the paper [5] entitled "Experimental evidence of non local hydrodynamic influence on the dynamic contact angle". Our conclusion can be taken, to a certain extent, as being just reciprocal, since it brings an evidence of the influence a local capillary length scale has on the whole hydrodynamics of the liquid bridge.

How to deal with this singularity? To the authors' knowledge, the numerical works published so far either ignore it, by using discretization methods which filter it implicitly in a scheme- and mesh-dependent way, or regularize it without going further in the analysis of the consequences. All that has been commented at length in [6], and the main issue of this approach is presented now. It is assumed that physics must be regular at the continuous medium scale, the singularity revealing a deep physical problem whose hydrodynamic modeling is still unknown. Are there simple, but physically relevant, boundary conditions able to regularize the problem with temperature-dependent surface tension or viscosity considerations? The simplest that can be done, so far, is to regularize the capillary condition, and to reckon with the principle that Saint-Venant has invoked in linear elasticity: two different but statically equivalent ${ }^{1}$ distributions of contact forces acting on a surface portion of an elastic body have essentially the same effects beyond a large enough distance from the application zone. Our approach introduces a new free parameter, a length scale, with respect to which the convergence of the resulting numerical flows must be assessed. Two regularizations have been adopted to cancel smoothly the imposed vorticity at both extremities of the free surface:

(a) a fixed or passive regularization,

$$
\left.\left(\frac{\partial w}{\partial r}\right)\right|_{r=1}=-\left.M a\left(\frac{\partial \theta}{\partial z}\right)\right|_{r=1} f_{n}^{(p)}(z)
$$

where $f_{n}^{(p)}(z)$ is a polynomial, $f_{n}^{(p)}(z)=\left(1-z^{2 n}\right)^{2}, n$ being a positive integer. A measure of the filtering length, the distance over which the regularization acts, is defined by $\delta(n)$ such that $f_{n}^{(p)}(z=1-\delta(n))=0.95$, which leads to

$$
\delta(n)=1-0.0253^{1 / 2 n},
$$

of order $1 / n$ for large $n$. The stiffest regularization applied in this paper is $f_{13}^{(p)}(z)$, with $\delta(n=13)=0.132$. The $f_{n}^{p}(z)$ profiles have been given in $[6,7]$.

(b) a flow dependent or active regularization,

$$
\left.\left(\frac{\partial w}{\partial r}\right)\right|_{r=1}=-\left.M a\left(\frac{\partial \theta}{\partial z}\right)\right|_{r=1} f^{(a)}(\theta(r=1, z))
$$

This condition amounts to admit that the Marangoni number cannot be anymore constant in the neighborhood of the disks where strong thermal gradients are expected to occur. A phenomenological temperature dependence is then specified for the quantity $(\partial \sigma / \partial T) / \mu \kappa$. Such a condition is active since the temperature on the free surface is controlled by the flow. The function $f^{(a)}(\theta(r=1, z))$ is chosen to vanish exponentially at $z= \pm 1: f^{(a)}(\theta)=\exp \left[-\alpha\left(\theta_{\infty}-\theta\right) / \theta\right]$, with $\alpha$ a positive constant and $\theta_{\infty}$ the reduced temperature that is assigned by the flow on the free surface, "far" from the disks. The parameter $\alpha$ can be chosen, at any $M a$ value, so that both regularizations act on comparable filtering lengths.

For given $M a$ values, the convergence of the results has been followed with $n$, the passive regularization parameter. Two possibilities arise. First, there is only one stable steady flow regime. The maximum amplitude of its physical fields, for instance the vorticity which is the best sensor [7] when gravity is suppressed, grows up monotonically with $n$. In this case, the results can be considered as physically relevant when a quantitative convergence is achieved. The buoyant-capillary flows do converge in this fashion [6], and so do the pure capillary flows which are self-symmetric with respect to $\Pi$ at large $M a$ values, but with an extremely high computational cost for reaching the asymptotic states [7]. However, still in the pure capillary case and with small $M a$ values, another occurrence happens : asymmetrical stable

\footnotetext{
${ }^{1}$ that is with equal resulting forces and moments
} 
steady flows are allowed which also quantitatively converge with $n$. This is the case which is reported hereafter. Next, applying the active condition with an adequate value of $\alpha$ leads to close results, which is a good indication of their physical relevance.

\section{ABOUT THE NUMERICAL ASPECTS}

A complete insight into the flow dynamics requires to call for complementary numerical approaches, described in detail in [6]. All are based on the pseudospectral Chebyshev collocation method [8] for space derivatives evaluations, and on a usual second order finite difference scheme for time integration. Stringent criteria have been applied to assess the overall accuracy of the results. Correlated with a time marching procedure (which supplies only stable steady or oscillatory flows), specific tools (based on a continuation process) have been implemented for drawing, in the parameters space, the loci of the steady states, stable or not. By computing the leading eigenmodes [9] of the problem linearized in perturbations, Hopf, pitchfork or saddle-node bifurcations can be detected.

\section{RESULTS}

They are presented in four steps. The steady states which are self-symmetric by reflection about $\Pi$ are first described for their evolution with $n$, at $M a=100$. Next, the steady states (self-symmetric or not) are followed in $M a$, for $n=13$ and then for $n \leq 13$, and comparative results are given with the active regularization. The third section describes the asymmetrical fluid flow patterns. At last some elements are proposed on the physics of the self-symmetric states loss of stability.

\section{A. Evolution of the self-symmetric steady flows with $n$}

Owing to the geometry, the balance equations and boundary conditions, if $U$ is solution of the problem, then $U^{\prime}=\Xi(\boldsymbol{U})$ is also solution, $\Xi$ being the reflection symmetry about the mid-plane $\Pi$,

$$
\Xi:\left\{\begin{array}{l}
v^{\prime}(r,-z)=v(r, z) \\
w^{\prime}(r,-z)=-w(r, z) \\
\theta^{\prime}(r,-z)=\theta(r, z)
\end{array} .\right.
$$

Steady solutions, invariant through $\Xi$, are therefore expected to exist. Let us note them $\boldsymbol{U}_{s}$. They have been numerically obtained by time integration, either on a large $M a$ domain [10] with $n=1$, or at some Ma sampled values with $n>1$ [7]. These flows are essentially made of two counter-rotating cells, each one located on either side of $\Pi$ (Fig. 3 (a)). At small $\operatorname{Pr}$ and $M a$ values, the temperature is mainly governed by conduction, heat being driven from the central part of the free surface towards the solid planes (Fig. 3 (b)). The contour lines of the velocity radial and axial components are drawn in Figs. $3(\mathrm{c})$ and $(\mathrm{d})$. The strong slowing down $(n=13)$ of the velocity axial component at the arrival on the solid boundaries generates a jet-like incoming radial flow. These are the main features of the self-symmetric steady states which we are now going to follow with $n$, keeping $M a=100$.

Figs. 4 and 5 respectively show, for $n$ going from 1 to 13 , the normalized vorticity, $(1 / M a)(\partial w) /\left.(\partial r)\right|_{r=1}$, and the velocity $w(r=1, z)$, as functions of $z$, on the lower half-part of the free surface. One notes the expected increase with $n$ of the absolute maxima, as well as the migration of their location on the free surface $\left(z_{w_{r}}^{\max }\right.$ and $\left.z_{w}^{\max }\right)$ towards the solid boundaries $z= \pm 1$, shown in Fig. 6 against $\delta(n)$ (see relation 5). Noticing the logarithmic scale of the vertical axis, the migration is exponential with $n$. It leads to the satisfactory result that the maxima locations fall well within the liquid bridge.

\section{B. Ma-Evolution of the steady states structure for $n=13$}

Starting from the self-symmetric steady state obtained with a time stepping code, at $n=13$ and a small enough $M a$ value, a continuation curve in $M a$ is computed, and the $2 \mathrm{D}$ stability of the steady states encountered on the way is examined. The temperature at $z=0.327$ on the free surface is the continuation sensor. The resulting curve 
is displayed on Fig. 7. The self-symmetric steady states lose their stability via a sub-critical pitchfork transition located at a critical $M a$ value noted $M a_{0}^{c}$. Three successive saddle-node bifurcations then follow, on each of the two branches emerging from the pitchfork bifurcation, at the critical values $M a_{i}^{c},(i=1,3)$. One has respectively $\left(M a_{i}^{c}, i=0,3\right) \approx 105,57,1812,316$ for $n=13$. The states lying on the branch which originates at $M a_{i}^{c}$ on the upper (lower) side of the $U_{s}$ states branch (the solid line of Fig. 7) are noted $U_{i}\left(U_{i}^{\prime}\right.$ resp.).

\section{The loss of stability of the self-symmetric steady flows}

Let $\Psi$ be an eigenmode of the set of equations which are linear in perturbations about the self-symmetric flows. $U_{s}$. It can be shown that if $\Psi$ is solution of this problem, then $\Psi^{\prime}=\Xi(\Psi)$ and $\Psi^{\prime \prime}=\Gamma(\Psi)$ are also solutions, $\Gamma$ being defined by

$$
\Gamma:\left\{\begin{array}{l}
v^{\prime \prime}(r,-z)=-v(r, z) \\
w^{\prime \prime}(r,-z)=w(r, z) \\
\theta^{\prime \prime}(r,-z)=-\theta(r, z)
\end{array} .\right.
$$

Fig. 8 presents the most destabilizing eigenmode obtained for $n=13$ and $M a=M a_{0}^{c}$. It is steady and invariant by $\Gamma$. Then, the symmetry property $\Xi$ of the eigenproblem entails that the loss of stability of the $U_{s}$ states occurs via a pitchfork bifurcation. The steady states $U_{i}$ and $U_{i}^{\prime}$ which emerge on the new branches after this bifurcation are not anymore invariant by $\Xi$, but $\Xi$-symmetrical of each other, $U_{i}^{\prime}=\Xi\left(U_{i}\right)$.

\section{Asymmetrical steady flows}

A schematic bifurcation diagram is given in Fig. 9, together with the stream line patterns of some chosen states. On the solid and dotted lines are respectively the stable and unstable steady states, $U_{0}$ being therefore unstable, and the subsequent $U_{i}$ alternatively stable and unstable. Several stable flows coexist at $M a$ values greater than $M a_{1}^{c}$. The diagram is illustrated with the stream function pattern of (a) marginal states (noted $U^{M a_{i}^{c}}$ ) on its upper part, and (b) intermediate states (noted $U_{i}^{\prime M a}$ ) on its lower part. The state noted $U^{M a_{0}^{c}}$ is self-symmetric. The flows are all made of two counter-rotating cells, obviously of the same size for the $U_{s}$ flows, and more and more uneven going up on the branch order number at fixed $M a\left(\operatorname{see} U^{M a_{0}^{c}}, U_{1}^{\prime 100}\right.$ and $U_{1}^{\prime 500}, U_{3}^{\prime 500}$ ).

\section{Convergence with $n$ of the bifurcation map}

How does this sequence of bifurcations converge with $n$ ? Actually, the existence of the first subcritical bifurcation is the main feature, and the bifurcations have been searched for, with $n>1$, but only if they occur at $M a<1000$, or so. The so-obtained thresholds $M a_{i}^{c}(n)$ are plotted against $\delta(n)$ in Fig. 10. They go down to smaller and smaller $M a$ values as the filtering length $\delta(n)$ decreases, and the first two thresholds clearly converge.

It is worth pointing out that the asymmetrical stable steady states are, for the time being, practically almost unreachable by the usual time-marching procedures (instead of the continuation approach [6]) because of the extremely small slope the perturbation growth rate has with $M a$ about the threshold $M a_{0}^{c}(n)$. Fig. 11 displays the dimensionless growth rate of the most destabilizing eigenmode as a function of $M a$ about $M a_{0}^{c}(n=13)$. The dimensionless time necessary to get the perturbation amplitude amplified by $e$ is about 1000, and the access to settled states by typical time steps of $5 \times 10^{-4}$ requires dramatically long numerical experiments.

\section{Active regularization}

There is no reason to perform a so detailed analysis with the active regularization in the absence of any theoritical justification of our models and of any physical interpretation of the free parameters, $n$ or $\alpha$. Moreover, invoking the Saint-Venant principle, the difference between both these models (and possibly with the correct one) should not affect the flow much beyond a small region surrounding the junction of the free surface with the solid boundaries. The active stress condition is therefore simply used to check the qualitative relevance of the previous results, that is mainly the existence of the non-symmetrical stable steady flows. Giving the constant $\alpha$ the value 0.1 leads, for $\mathrm{Ma}=100$, to a 
filtering length close to $\delta(10)$, as can be seen in Fig. 12 where $f^{(a)}(\theta)$ is plotted as a function of $z$ and compared with $f_{n}^{(p)}(z), n=1,5,10$. The stable steady flows were computed (by a time-marching procedure) at $M a=1,10,50,100$ and 500. A steady asymmetrical state emerges only for $M a=500$ where the flow pattern, presented by the stream function contour lines of Fig. 13, looks very much like the one labeled $\boldsymbol{U}_{1}^{\prime 500}$ in Fig. 9.

\section{Asymmetrical fluid flow structures}

\section{Flow patterns}

The stream lines pattern of the marginal steady states are on Fig. 9 and the profiles of their free surface velocity are displayed in Fig. 14. The asymmetry is less pronounced on the free surface than in the flow core. Following the bifurcation curve, the location on the free surface where the axial velocity cancels moves, but only slightly, up and down about $z=0$, without correlation with the cell size evolution (see the $M a_{3}^{c}$ case for instance). But a close inspection of the smallest cell of the $\boldsymbol{U}^{M a_{2}^{\iota}}$ and $\boldsymbol{U}^{M a_{3}^{c}}$ flows in Fig. 9 shows that each one has a long tail stretching pinched between the largest cell and the free surface, a structure which is clearly exhibited by the vorticity lines drawn in Fig 15 for the $U^{M a_{3}^{c}}$ case. In contrast with the bulk flow, the vorticity on the free surface is mostly symmetrical: the convection is not strong enough in this fluid of low Prandtl number to damp the symmetrical contribution of the imposed lateral heat flux.

Thus, measurements of superficial fields of flows occuring in liquid metals cannot be considered as reliable as regards the flow patterns.

\section{Free surface shape}

The knowledge of the flow pressure field allows us to solve the Laplace equation and to reconstruct the free surface shape, fixing the radius and letting free the deflection angle. The material properties have been given typical values corresponding to $S i$, leading to a small capillary number $C a=5.6 \times 10^{-6} \mathrm{Ma}$. The input data are listed in the caption of Fig. 16 where the shapes are reported for some flows of Fig. 9. In all cases the magnitude of the deflection is less than 1\%, justifying, a posteriori, that the free surface has been taken as non-deformable, with the assumption that this deflection has a negligible feed-back effect on the flow. The symmetry loss amplifies the magnitude of the free surface deflection and alters its shape : almost straight in the self-symmetric case $\left(\boldsymbol{U}^{M a_{0}^{c}}\right)$ it becomes mostly sinuous in the others, the largest cell hollowing the free surface by mass transfer from the lower to the upper part of the cavity. The free surface shape is therefore a good witness of the bulk flow structure.

\section{Remarks}

The side-heated liquid bridge is by far much less documented than the half-zone problem. The results obtained in the latter configuration may be non-relevant for describing the first transitions from 2D to 3D steady and unsteady flows of the laterally heated configuration. Mentioned for the first time as being steady and stable axisymmetrical solutions of the side-heated liquid bridge, the above described asymmetrical states resemble the azimuthal snapshots of the 3D unsteady numerical results obtained by [11] and, more recently, by [4] in comparable conditions $(\operatorname{Pr}=0.04)$. This latter paper shows a steady axisymmetric numerical flow which seems self-symmetric about the mid-plane and becomes, with increasing $M a$, three-dimensional and, next, time-dependent. Only the existence of the 2D $\rightarrow$ 3D transition is mentioned whose threshold seems to lie in between $M a_{0}^{c}(n=13)$ and $M a_{3}^{c}(n=13)$ (see Fig. 10). The threshold of the second transition is announced at $M a=355 \pm 90$, that is around $M a_{3}^{c}(n=13)$. At last, a recent compilation of the experimental thresholds to unsteady flows is proposed in [12], with most of the data coming from the half-zone problem. For $\operatorname{Pr}=0.01$ the thresholds lie in $M a \in[80,200]$, with large error bars since critical $M a$ values are not easy to measure. All that clearly indicates that an important part of the liquid bridge dynamics occurs in a quite reduced domain located about $M a \simeq 150$.

\section{Physics of the symmetrical flow loss of stability}

What can be said about the physical instability mechanisms that are responsible for the loss of stability of the symmetrical flows? The local and global rates of change of the kinetic energy are evaluated for the leading eigenmode 
of the equations linearised in perturbations about the symmetrical states $U_{s}$. The local rate can be decomposed as

$$
\frac{\partial}{\partial t}\left(\frac{(\delta u)^{2}}{2}\right)=T_{I^{\delta v ; v, r}}+T_{I^{\delta v ; v, z}}+T_{I^{\delta w ; w, r}}+T_{I^{\delta w ; w, z}}-T_{D}+T_{M}+E
$$

where the last term $E$ contains all the contributions of which the volume integral cancels. The other terms are successively

$$
\begin{gathered}
T_{I^{\delta v ; v, r}}=-(\delta v)^{2} \frac{\partial v}{\partial r}, T_{I^{\delta v ; v, z}}=-\delta v\left(\delta w \frac{\partial v}{\partial z}\right), \\
T_{I^{\delta w ; w, r}}=-\delta w\left(\delta v \frac{\partial w}{\partial r}\right), T_{I^{\delta w ; w, z}}=-(\delta w)^{2} \frac{\partial w}{\partial z}, \\
T_{D}=\operatorname{Pr}(\nabla \times \delta u)^{2}, T_{M}=\operatorname{Pr} \delta w \frac{\partial(\delta w)}{\partial r} \delta(r-1),
\end{gathered}
$$

where $\delta \boldsymbol{u}=\delta v \boldsymbol{e}_{r}+\delta w \boldsymbol{e}_{z}$ is the velocity perturbation and $\delta($.$) the Dirac function. The global rate reads then$

$$
\dot{E}_{c}=\frac{\partial}{\partial t}\left(\int_{0}^{1} \int_{-1}^{1} \frac{(\delta u)^{2}}{2} r d r d z\right)=-D+M+I^{\delta v ; v, r}+I^{\delta v ; v, z}+I^{(\delta w ; w, r)}+I^{(\delta w ; w, z)},
$$

where all terms are volume integral, such as $D=\iint T_{D} r d r d z$, and so on. All the data quoted here have been obtained with $n=13$.

The surface of Figs. $17(\mathrm{a}-\mathrm{b})$ and $18(\mathrm{a}-\mathrm{d})$ gives the perturbation local power $\frac{\partial}{\partial t}\left(\frac{(\delta \boldsymbol{u})^{2}}{2}\right)$ as a function of $r \in[0,1]$ and $z \in[-1,0]$, i.e. in the lower half part of the liquid bridge, for $M a=110$, slightly above $M a_{c}^{0}$. Two regions are to be noticed, one (denoted $\mathcal{R}_{0.5}$ ) where the power has an absolute maximum, near the mid-plane $\Pi$, at about $r=0.5$, and the second (denoted $\mathcal{R}_{1}$ ), still about the mid-plane, but closer to the free surface, where the power has a smaller maximum. The energy growth rate of the symmetrical states occurs therefore "far" from the junction of the free surface with the solid boundaries.

The base state vorticity reaches a maximum close to $\mathcal{R}_{0.5}$, as shown by Fig. 17 (a). The action of the term $E$ (Fig 17 (b)), of zero global contribution, is to transfer power from $\mathcal{R}_{0.5}$ (negative) to $\mathcal{R}_{1}$ (positive isolines). The leading contributions to the perturbation power come from the four convective terms $T_{\Gamma \delta v ; v, z}, T_{\Gamma \delta w ; w, z}, T_{\Gamma \delta w ; w, r}$ and $T_{I^{\delta v ; v, r}}$. Some of their isolines are superimposed on the local power distribution, in Figs. 18 (a), (b), (c) and (d) respectively. All but $T_{I^{\delta w ; w, r}}$ have their maximum localized in $\mathcal{R}_{0.5}$. From (a) and (d) one notes that the $T_{I^{\delta v ; v, z}}$ and $T_{I^{\delta v ; w, r}}$ terms compensate themselves, and $T_{I^{\delta w ; w, z}}$ in (b) is significantly more important than its $(\mathrm{c})$ partner $T_{I^{\delta w ; w, r}}$.

The global quantities are now considered about $M a_{0}^{c}(n=13)$ as functions of $M a$. In Fig. 19 are plotted all terms in (6) normalized by $D$. As already indicated, the growth of $\dot{E}_{c}$ with $M a$ is extremely small. The most contributing term, $I^{(\delta w ; w, r)}$, decreases with $M a$, while the next one, $I^{\delta v ; v, z}$ increases. One notes also that the main term of the Figs. 18, namely $T_{I^{\delta w ; w, z}}$ in (b), has an integrated contribution of modest importance, increasing however with $M a$, from negative to positive supplies to $\dot{E}_{c}$, respectively before and after the transition.

\section{CONCLUSIONS}

An explicit smooth matching of the boundary conditions at the contact free surface/solid boundary and a 2D linear stability analysis of the steady axisymmetric thermocapillary flows have led to point out the sensitivity of the flow global structure to local capillary contributions, in a side-heated liquid metal bridge $(\operatorname{Pr}=0.01)$. The coexistence of multiple stable steady states, self-symmetric or not with respect to the mid-plane, is predicted, at values of $M a$ which turn out to be small at the capillary length scale convergence, and close to the thresholds mentionned elsewhere for the onset to unsteadiness. An important part of the side-heated liquid bridge dynamics happens in a reduced $M a$ domain, located about $M a \simeq 150$.

Acknowledgments - The numerical calculations were carried out on the vectorial computers of the CRI (PARIS-SUD XI University) and IDRIS (CNRS) centers. 


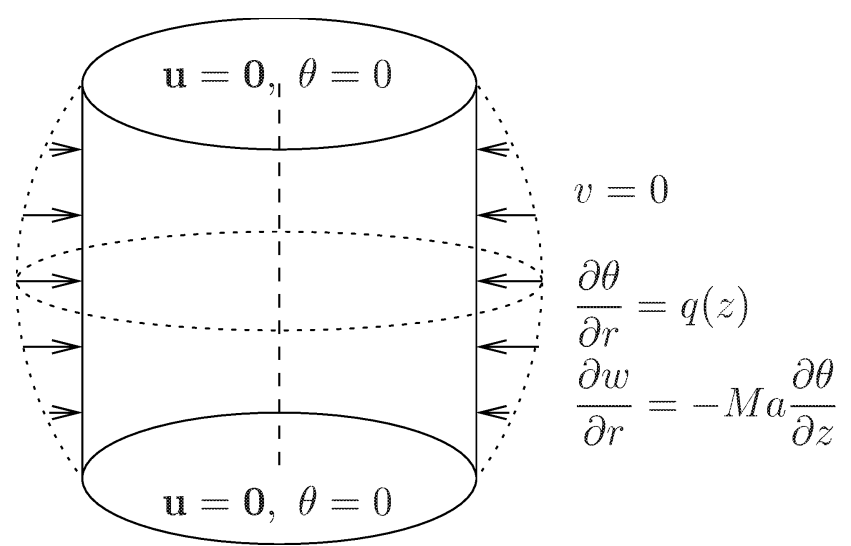

FIG. 1. Sketch of the side heated floating zone with the boundary conditions.

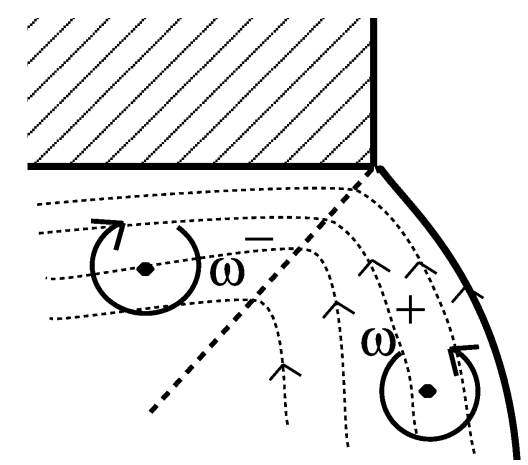

FIG. 2. Vorticity singularity at the junction free surface/solid boundary.
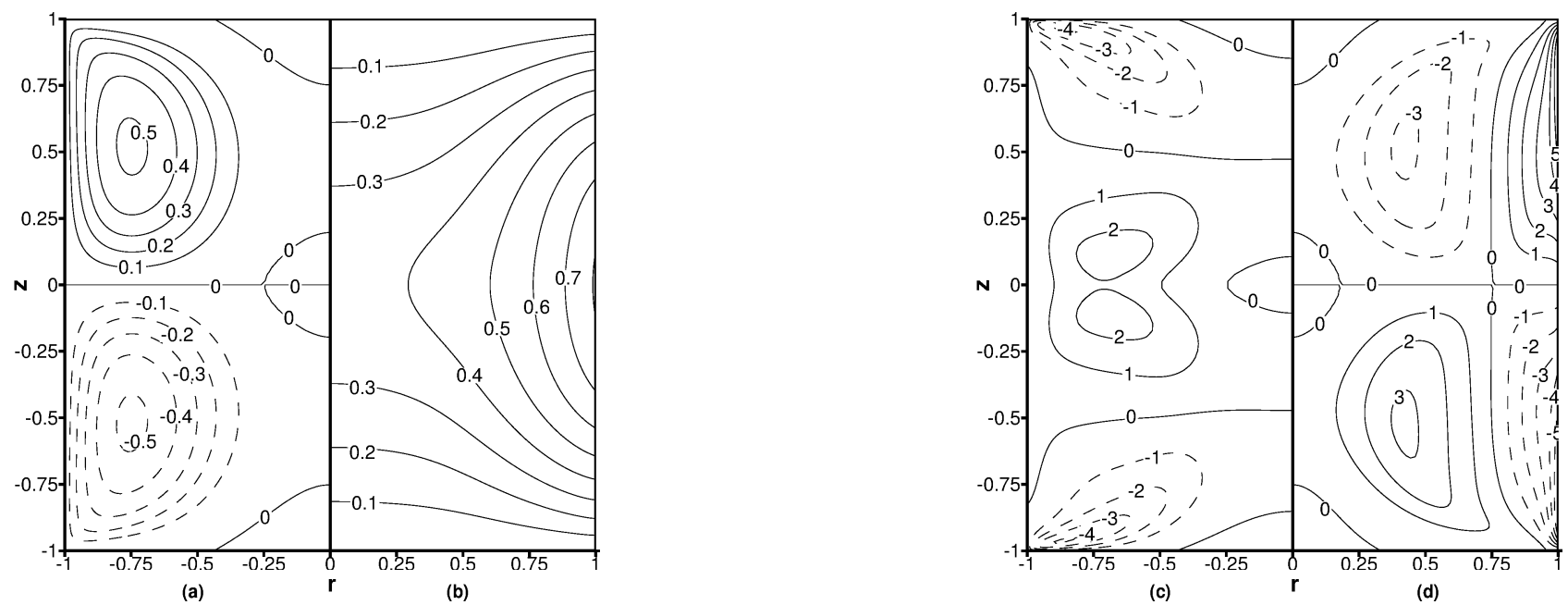

FIG. 3. (a) stream function, (b) temperature, (c) radial and (d) axial velocity components of the symmetrical steady state at $M a=100$. 


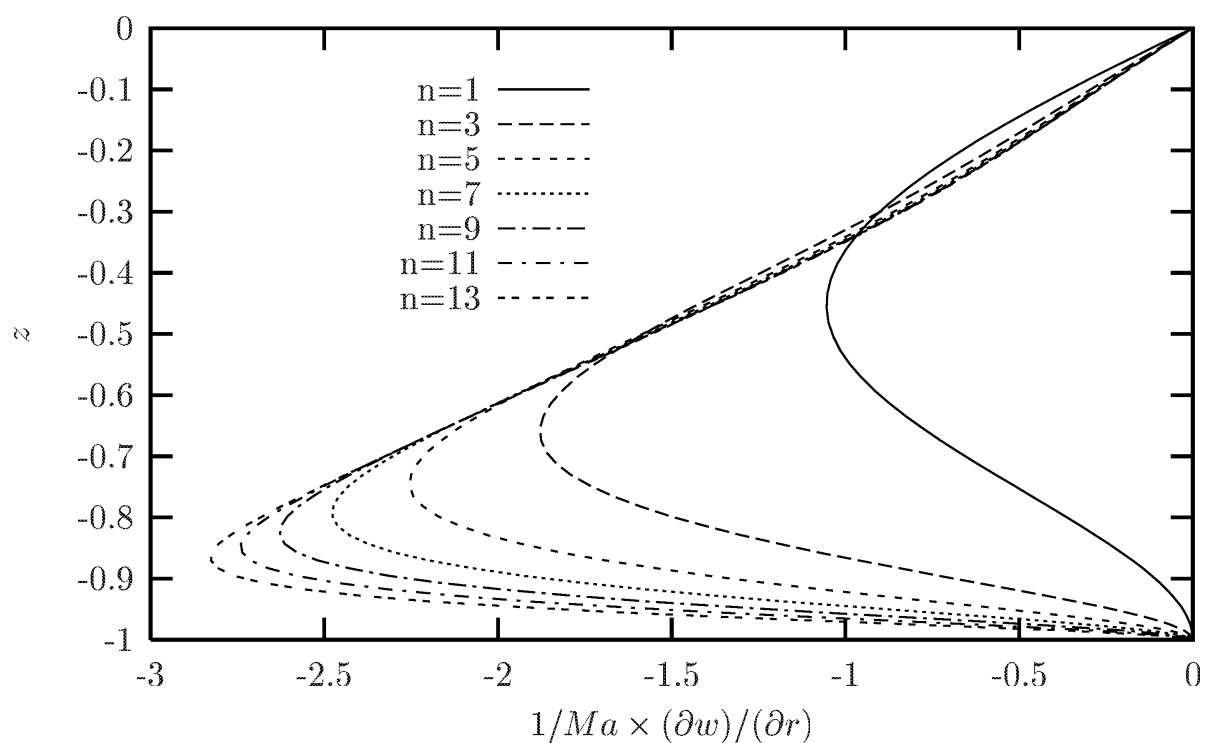

FIG. 4. Normalized vorticity on the free surface lower half, for increasing steepnesses $n$ of the passive regularization function, at $M a=100$.

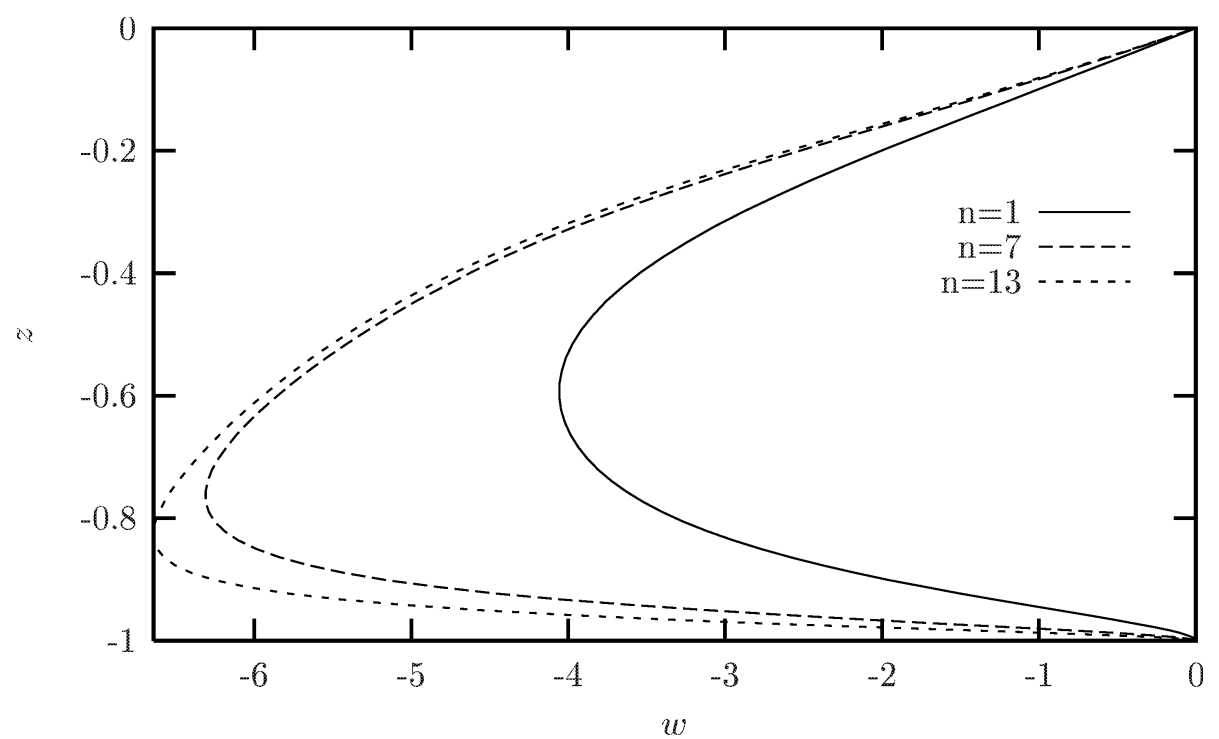

FIG. 5. Axial velocity on the free surface lower half, for three $n$ values, at $M a=100$. 


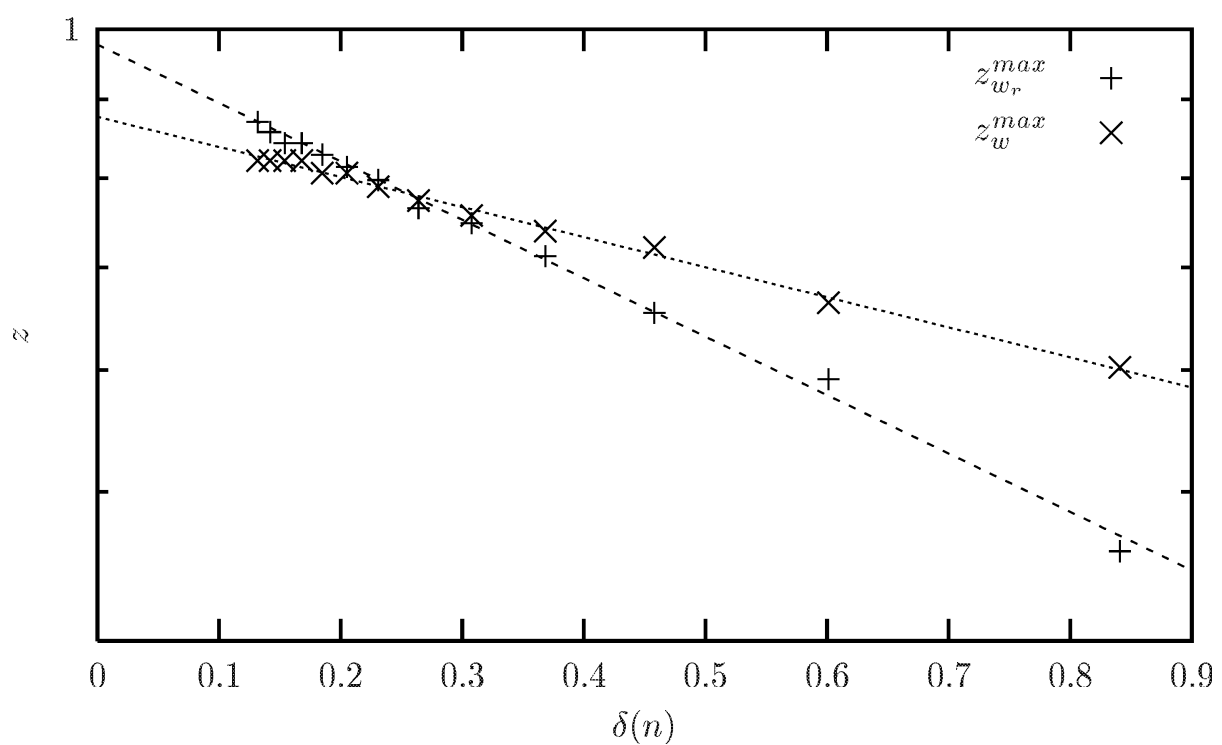

FIG. 6. Position, on the free surface, of the extrema of the axial velocity and stress as a function of the filtering length, at $M a=100$.

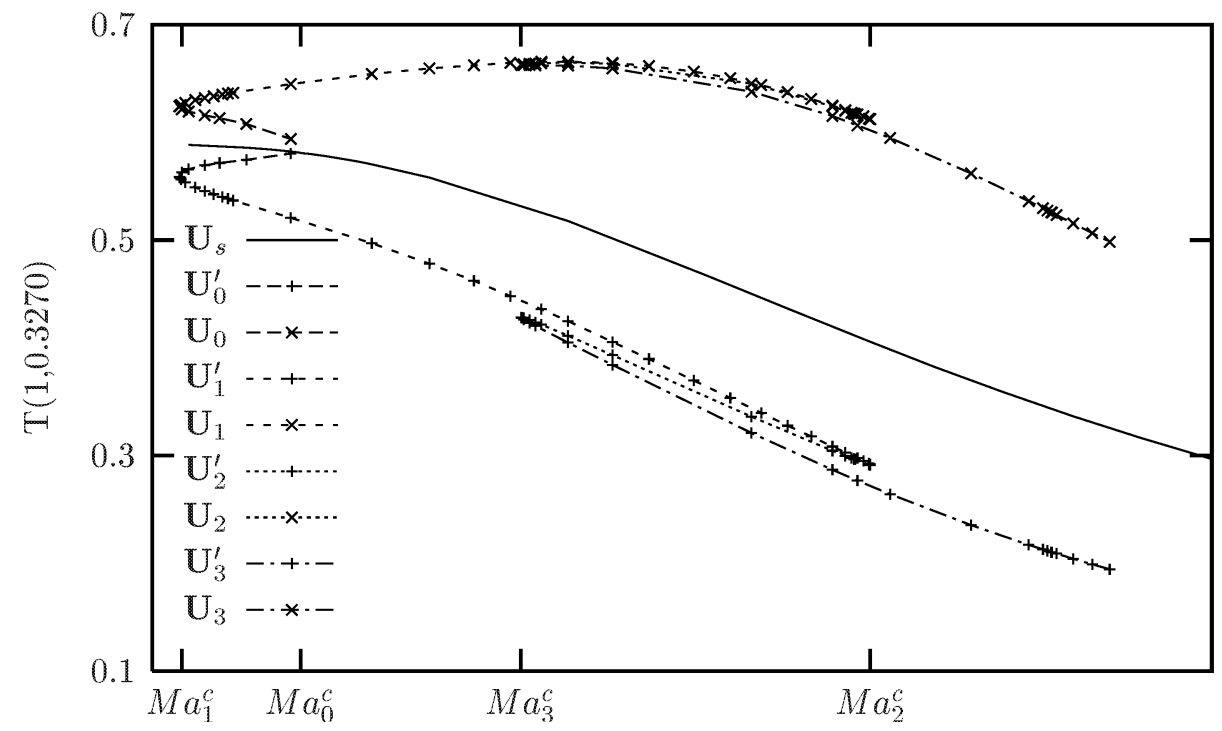

FIG. 7. Continuation curve of the temperature on the free surface at $z=0.3270$ as a function of the Marangoni number, for $n=13$. 

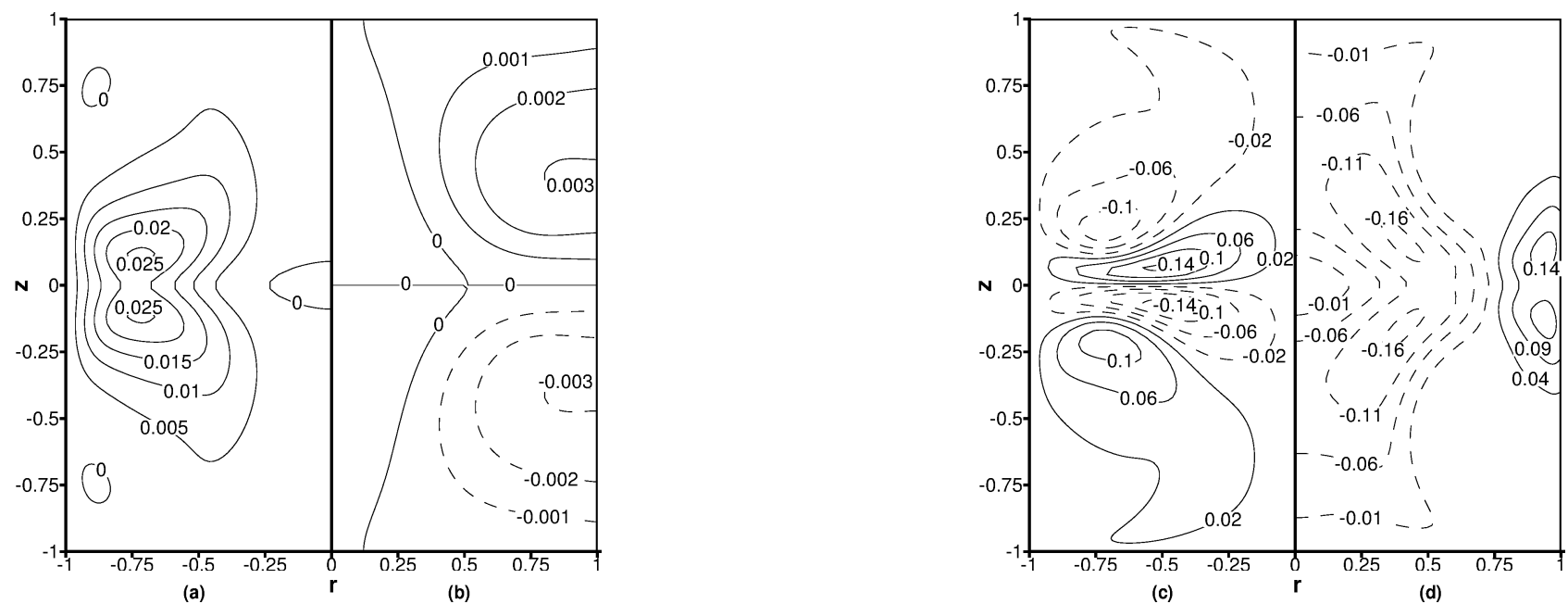

FIG. 8. (a) stream function, (b) temperature, (c) radial and (d) axial velocity components of the dominant eigenmode at $M a_{0}^{c}$. 


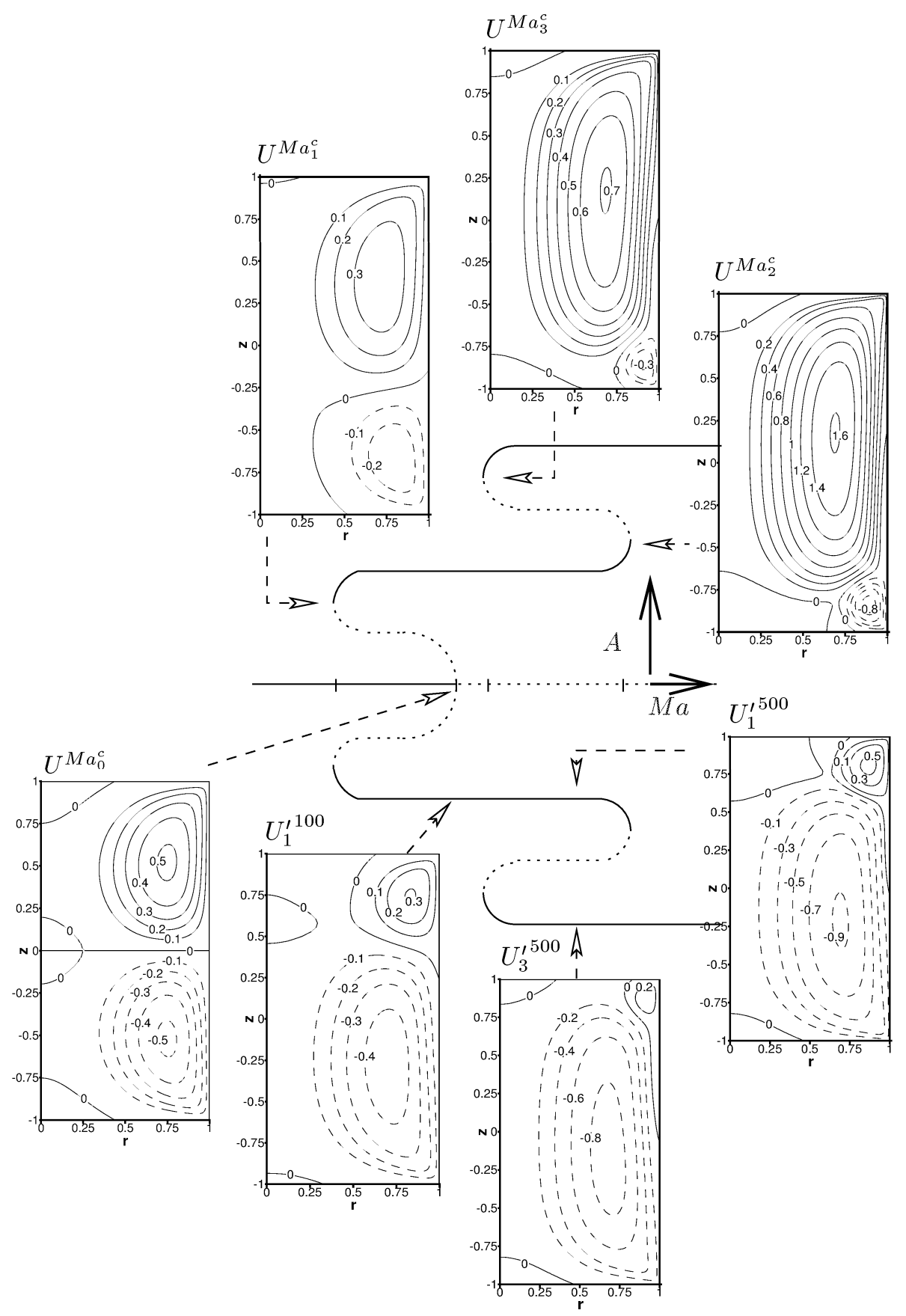

FIG. 9. Schematic bifurcation diagram and stream lines of quasi-critical steady states (on the upper part) and stable steady flows at $M a=100$ and $M a=500$ (on the lower part). The axis $A$ bears an amplitude. The stable (unstable) steady states branches are represented by solid (dashed) lines. The patterns have been computed with $f_{13}^{(p)}(z)$. 


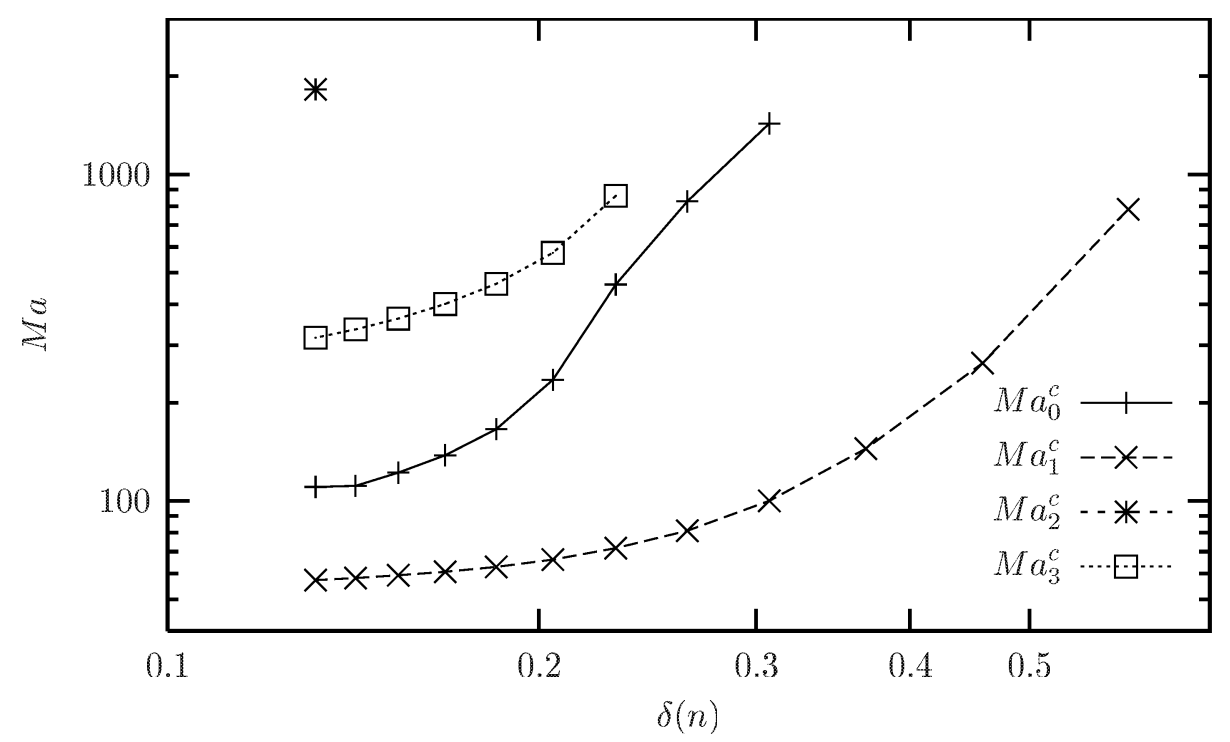

FIG. 10. Evolution of the critical Marangoni numbers with the filtering length $\delta(n)$.

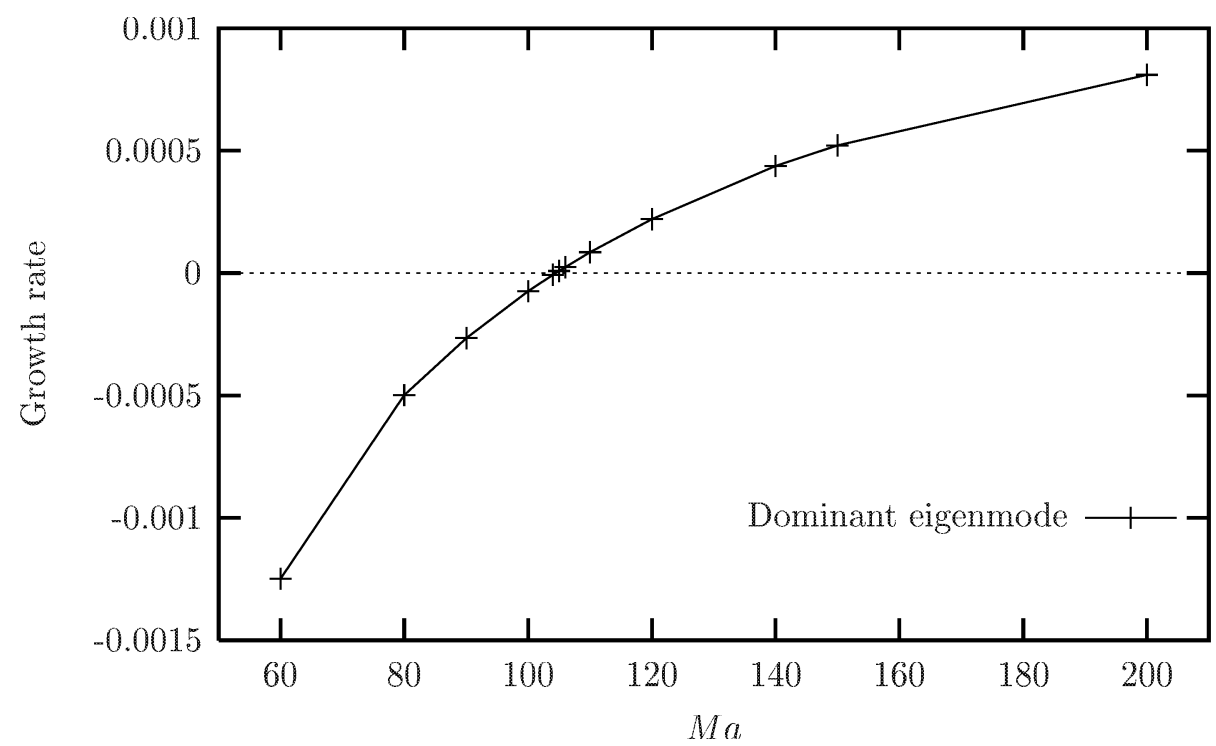

FIG. 11. The dimensionless eigenvalue of the most destabilizing mode of the symmetrical steady flows as function of $M a$ about $M a_{0}^{c}$. 


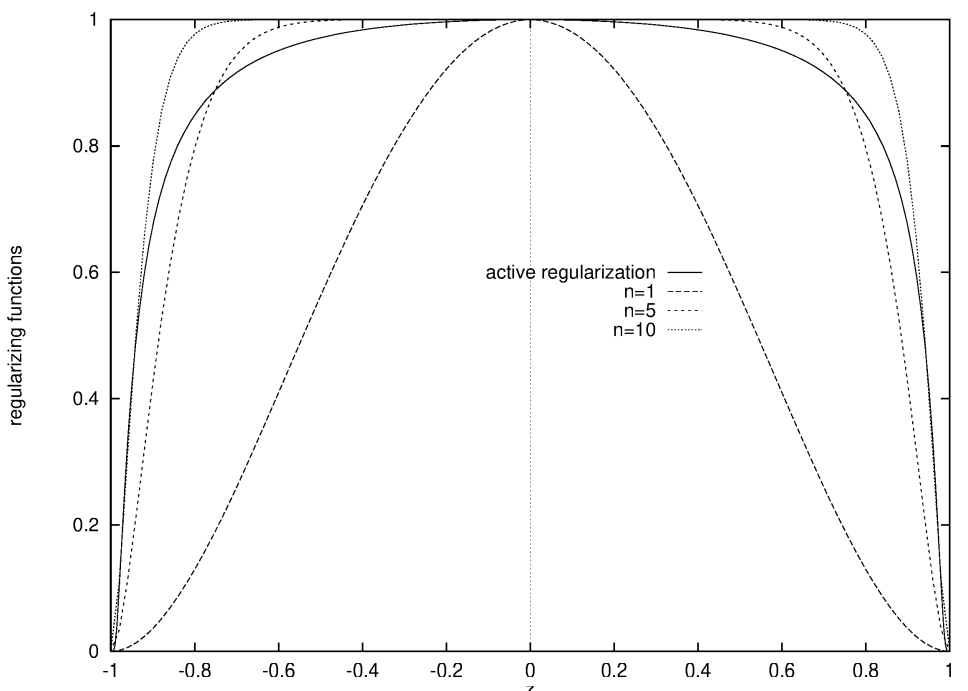

FIG. 12. The active regularization $f^{(a)}(\theta)$ calculated at $M a=100$, with $\alpha=0.1$, compared with $f_{n}^{(p)}(z), n=1,5,10$.

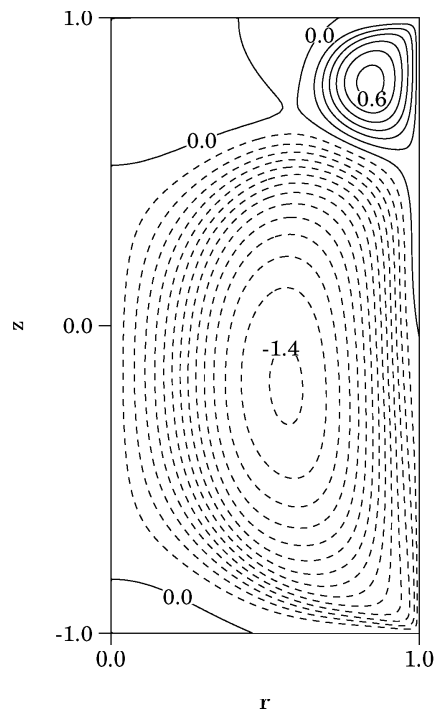

FIG. 13. Stream function lines of the stable steady state obtained with the active regularization $f^{(a)}(\theta)$ computed with $\alpha=0.1$, at $M a=500$. 


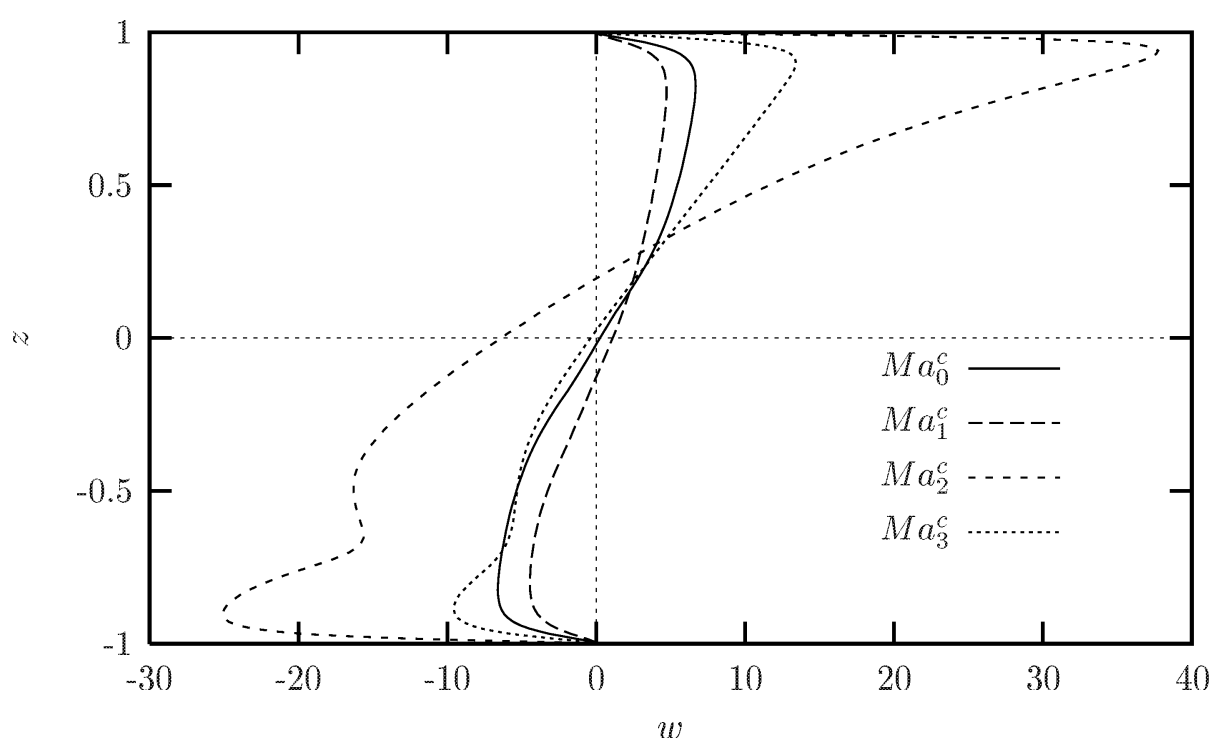

FIG. 14. Axial velocity on the free surface of the quasi-critical states shown on Fig. 9.

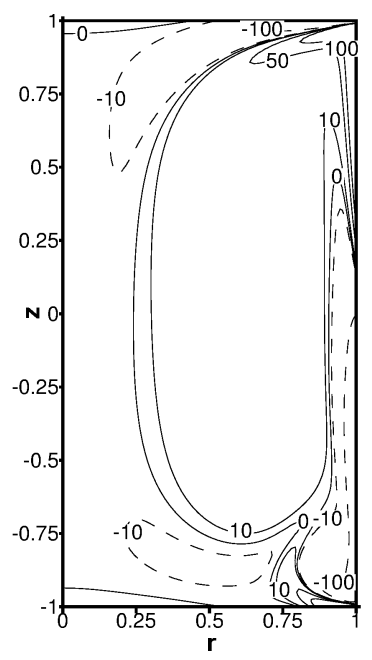

FIG. 15. Vorticity of the $U^{M a_{3}^{c}}$ state. 


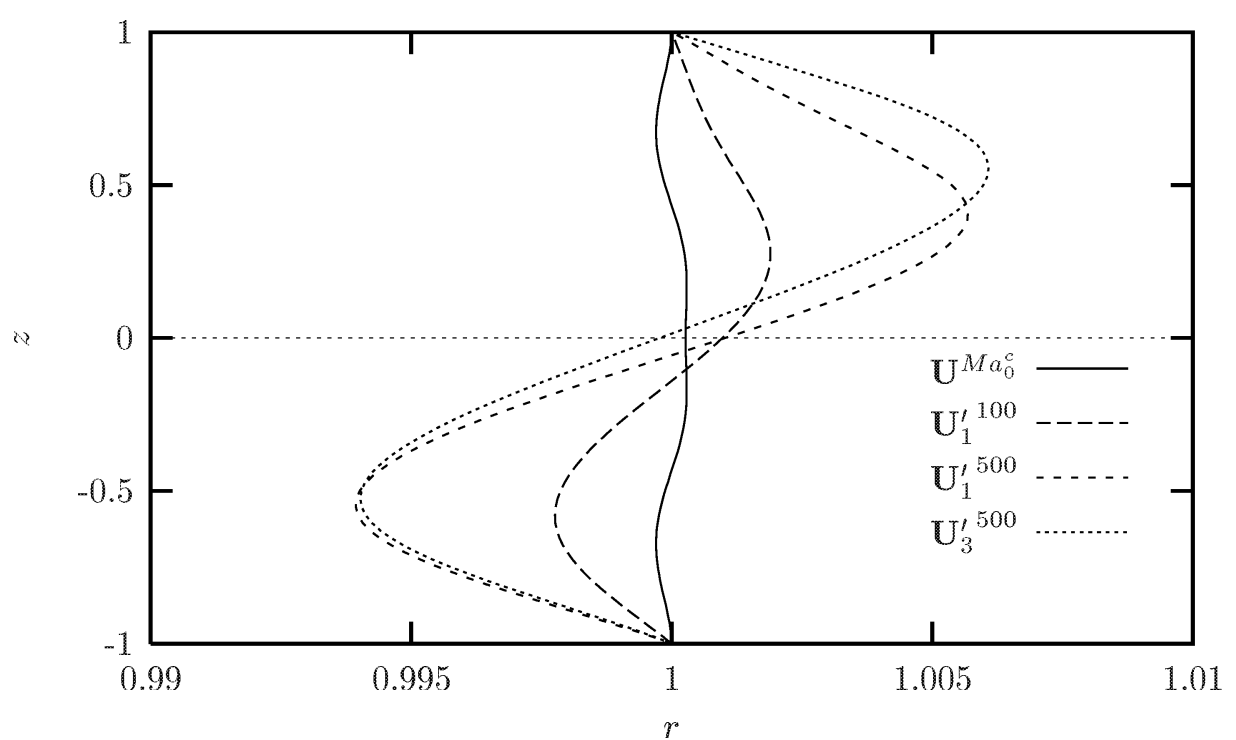

FIG. 16. Shape of the free surface for the flows $U^{M a_{0}^{c}}, U_{1}^{\prime 100}, U_{1}^{\prime 500}$ and $U_{3}^{\prime 500}$ of Fig. 9. The computations have been performed with $\mu=8.8 \times 10^{-4} \mathrm{~kg} / \mathrm{m} . \mathrm{s}, \kappa=2.75 \times 10^{-5} \mathrm{~m}^{2} / \mathrm{s}, \sigma=0.87 \mathrm{~N} / \mathrm{m}$ and $R=0.5 \mathrm{~cm}$. The capillary number $C a=\frac{\delta \sigma}{\sigma\left(T_{m}\right)}$ is equal to $5.6 \times 10^{-6} \mathrm{Ma}$. 


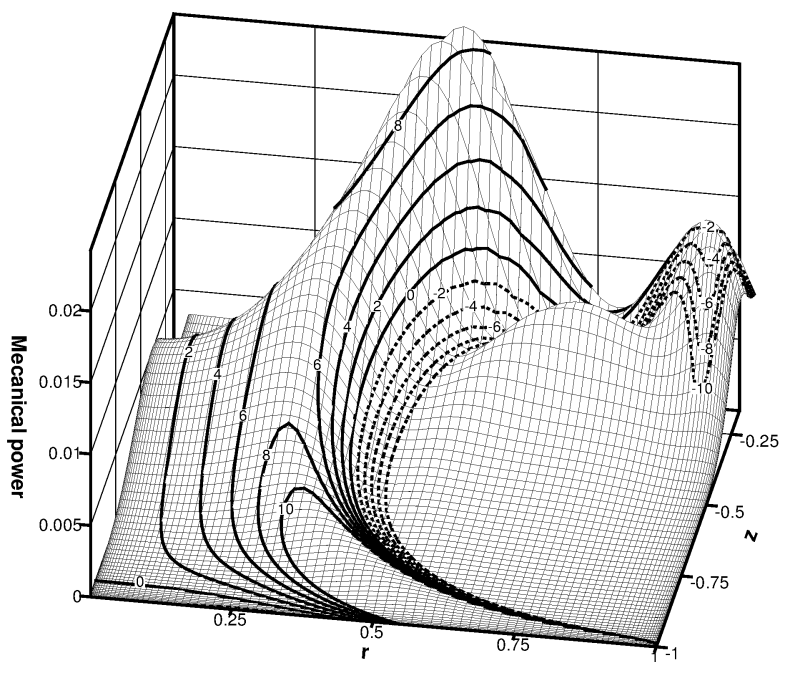

(a)

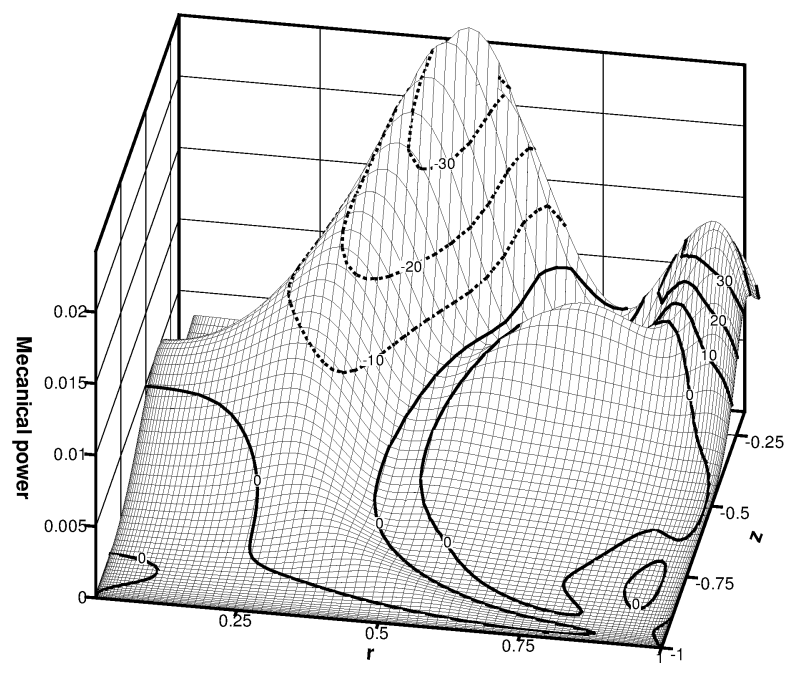

(b)

FIG. 17. The perturbation local power $\frac{\partial}{\partial t}\left(\frac{(\delta \boldsymbol{u})^{2}}{2}\right)$ and isolines of (a) the steady state vorticity, (b) $E$. 


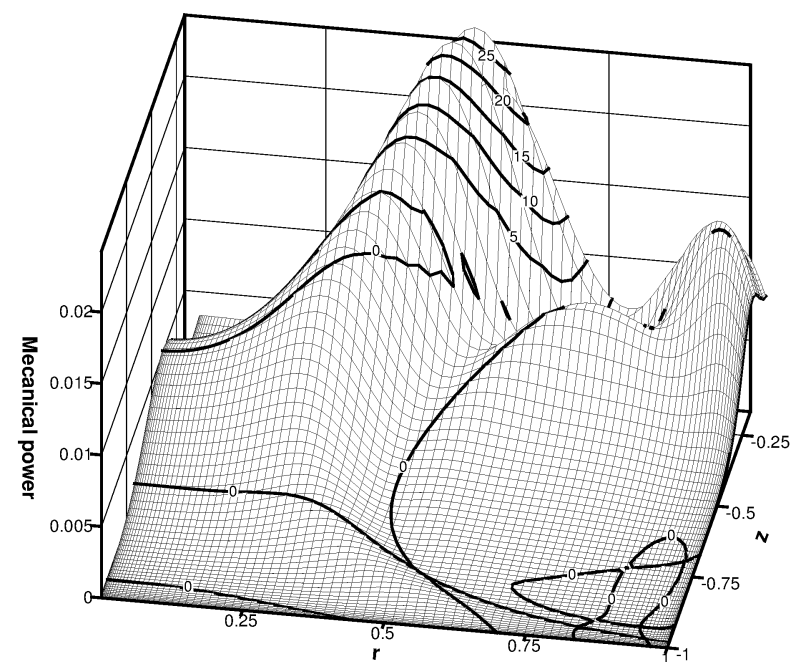

(a)

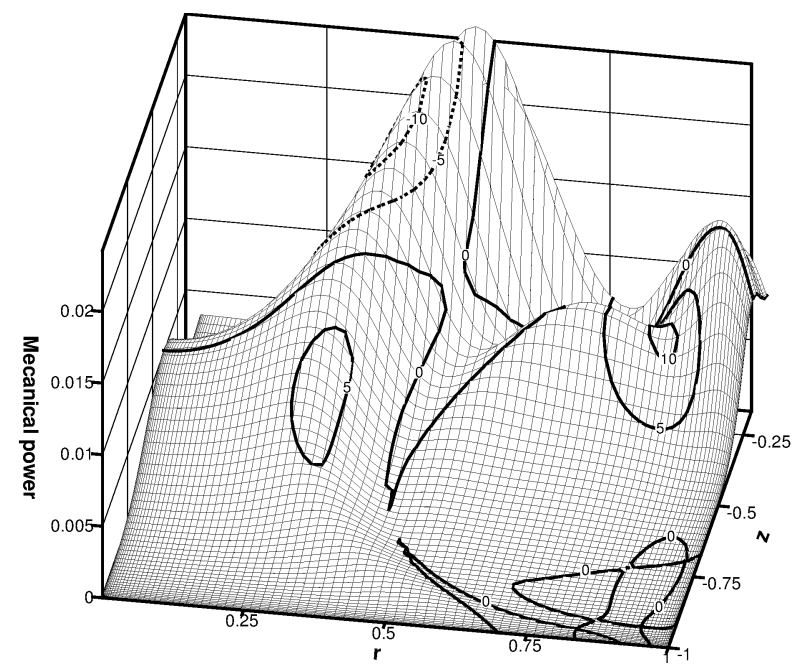

(c)

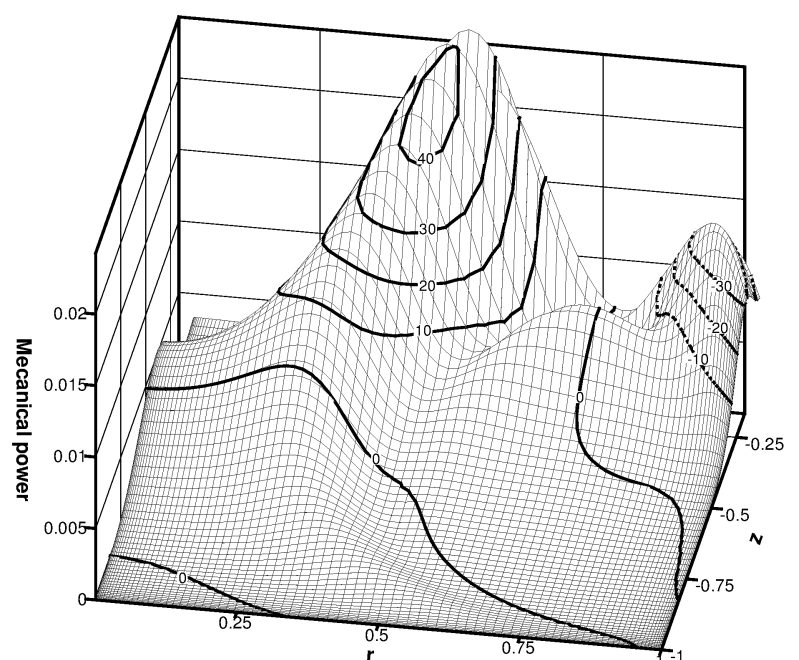

(b)

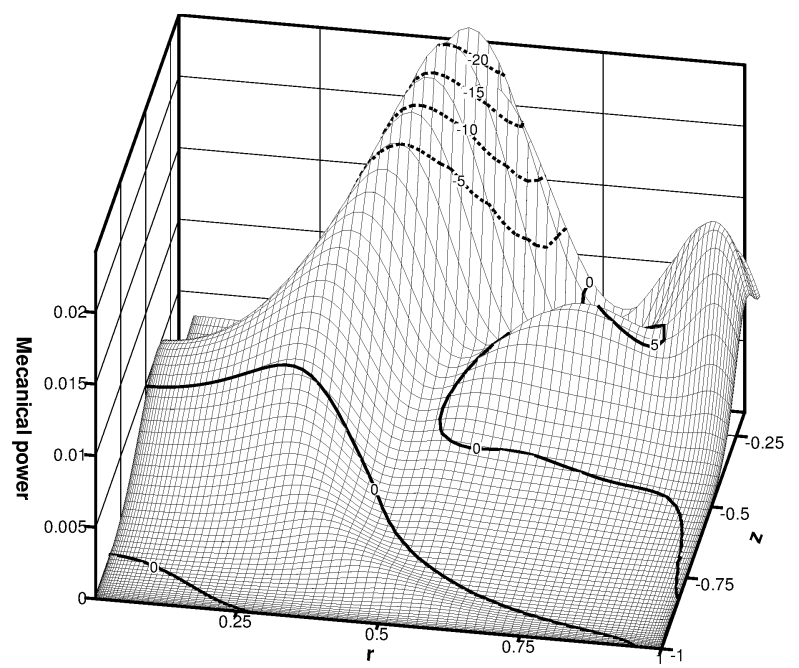

(d)

FIG. 18. The perturbation local power $\frac{\partial}{\partial t}\left(\frac{(\boldsymbol{\delta} \boldsymbol{u})^{2}}{2}\right)$ as function of $r \in[0,1]$ and $z \in[-1,0]$, for $M a=110>M a_{c}^{0}$, with some isolines of (a) $T_{I}^{\delta v ; v, z}$, (b) $T_{I}^{\delta w ; w, z}$, (c) $T_{I}^{\delta w ; w, r}$, (d) $T_{I}^{\delta v ; v, r}$. 


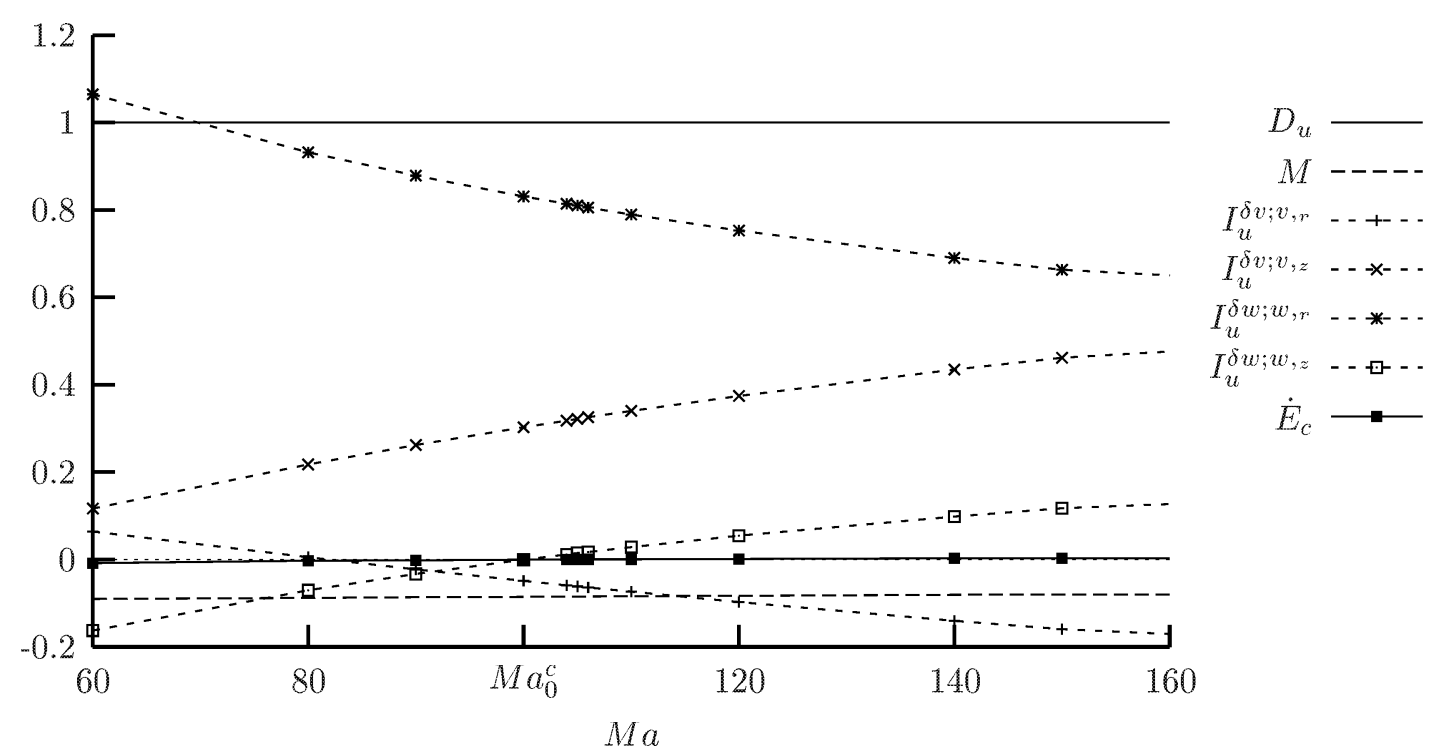

FIG. 19. Global power balance of the most destabilizing mode of the symmetrical steady flows as function of $M a$ about $M a_{0}^{c}$. 
${ }^{1}$ R. Emeis, "Tiegelfreies Ziehen vom Silicium-Einkristallen", Z. Naturforsch. A 9, 67 (1954).

${ }^{2}$ G. Müller, "Convection and inhomogeneities in crystal growth from the melt", in H.C. Freyard (Ed.), Crystals: Growth, Properties and Applications 12, Springer, Berlin, 1988.

${ }^{3}$ M.M. Rao and W. Shyy, "Moving boundary computation of the float-zone process", Int. J. Numer. Methods Eng 40,1231 (1997).

${ }^{4}$ A. Cröl, Th Kaiser, M. Schweizer, A.N. Danilewsky, S. Lauer, A. Tegetmeier and K.W. Benz, "Floating-zone and floatingsolution-zone growth of GaSb under microgravity", J. Crystal Growth 191, 365 (1998).

${ }^{5}$ T.D. Blake and M. Brake, Y.D. Shikhmurzaev, "Experimental evidence of nonlocal hydrodynamic influence on the dynamic contact angle", Phys. Fluids 11(8), 1995 (1999).

${ }^{6}$ E. Chénier, C. Delcarte and G. Labrosse, "Stability of the axisymmetric buoyant-capillary flows in a laterally heated liquid bridge", Phys. Fluids 11(3), 527 (1999).

${ }^{7} \mathrm{G}$. Kasperski and G. Labrosse, "On the numerical treatment of viscous singularities in wall-confined thermocapillary convection", Phys. Fluids 12(11), 2695 (2000).

${ }^{8}$ C. Canuto, M.Y. Hussaini, A. Quarteroni and T.A. Zang, Spectral Methods in Fluid Dynamics, Springer-Verlag Series in Computational Physics (Springer, Berlin, 1988)

${ }^{9}$ J. Goldhirsch, S.A. Orszag and B.K. Maulik, "An efficient method for computing leading eigenvalues and eigenvectors of large asymmetric matrices", J. Sci. Comput. 2, 33 (1987).

${ }^{10}$ G. Kasperski, A. Batoul and G. Labrosse, "Up to the unsteadiness of axisymmetric thermocapillary flows in a laterally heated liquid bridge", Phys. Fluids 12(1), 103 (2000).

${ }^{11}$ J. Baumgartl, M. Gewalt, R. Rupp, J. Stierlen and G. Müller, "The use of magnetic fields and microgravity in melt growth of semiconductors: a comparative study", Proc. 7th European Symposium on Materials and Fluid Sciences in microgravity, Oxford, UK, ESA SP-295, 47 (1990)

${ }^{12}$ Y.K. Yang and S. Kou, "Temperature oscillation in a tin liquid bridge and critical Marangoni number dependency on Prandtl number", J. Crystal Growth 222, 135 (2001). 